\title{
A simplistic computational procedure for tunneling splittings caused by proton transfer
}

\author{
Denis S. Tikhonov ${ }^{1,2}$ (D) \\ Received: 20 July 2021 / Accepted: 7 October 2021 / Published online: 27 October 2021 \\ (c) The Author(s) 2021
}

\begin{abstract}
In this manuscript, we present an approach for computing tunneling splittings for large amplitude motions. The core of the approach is a solution of an effective one-dimensional Schrödinger equation with an effective mass and an effective potential energy surface composed of electronic and harmonic zero-point vibrational energies of small amplitude motions in the molecule. The method has been shown to work in cases of three model motions: nitrogen inversion in ammonia, single proton transfer in malonaldehyde, and double proton transfer in the formic acid dimer. In the current work, we also investigate the performance of different DFT and post-Hartree-Fock methods for prediction of the proton transfer tunneling splittings, quality of the effective Schrödinger equation parameters upon the isotopic substitution, and possibility of a complete basis set (CBS) extrapolation for the resulting tunneling splittings.
\end{abstract}

Keywords Tunneling · Vibrations · Large amplitude motions · Quantum chemistry

\section{Introduction}

Large amplitude motions (LAM) are ubiquitous in most molecular systems, they are responsible for conformational interconversion and even for chemical reactions, such as in the cases of tautomerization [1-5]. One of the most prominent LAM manifestation is the tunneling splitting of the ground vibrational state, whenever a motion between equivalent minima is involved. Such splittings can be observed, for instance, using microwave (MW) or millimeter wave (MMW) rotational spectroscopy [6-15]. A knowledge of the LAM and of its characteristics can be helpful for the interpretation of the experimental data $[11,14,16,17]$.

An important chemical reaction, which is also a LAM, is the proton transfer via the Grotthuss mechanism [1, 3 , $6-9,15]$ :

$\mathrm{R}-\mathrm{O}-\mathrm{H} \cdots \mathrm{O}-\mathrm{R}^{\prime} \leftrightharpoons \mathrm{R}-\mathrm{O} \cdots \mathrm{H}-\mathrm{O}-\mathrm{R}^{\prime}$.

Denis S. Tikhonov

denis.tikhonov@desy.de

1 Deutsches Elektronen-Synchrotron DESY, Notkestr. 85, Hamburg 22607, Germany

2 Institute of Physical Chemistry, Christian-Albrechts-Universi tät zu Kiel, Max-Eyth-Str. 1, Kiel 24118, Germany
The canonical systems with such reactions are malonaldehyde (MA) and formic acid dimer (FAD) shown in Fig. 1. MA has a large tunneling splitting of $21 \mathrm{~cm}^{-1}$, which is well known from experiments for more than 20 years $[6,8]$. FAD is a system investigated theoretically for a long time $[18,19]$, but the experimental tunneling splitting (approx. $0.01 \mathrm{~cm}^{-1}$ ) was obtained only recently $[9,15,20,21]$.

Both these systems have been thoroughly investigated using various theoretical approaches. The low dimensional treatment of proton transfer and some of the coupled motion have been shown to give reasonable splitting estimates for MA [22], while for FAD such applications were less successful $[23,24]$. Applications of the reaction surface Hamiltonian (RSH) models [25-28] and of anharmonic normal mode-based treatments [18, 29-33] have yielded in good results for both MA and FAD. As expected, the full-dimensional quantum Monte-Carlo simulations provide results of the experimental quality for MA [29, 34, 35]. For MA and FAD, instanton approaches have been shown to produce accurate results [36-40].

In this paper, we present a modified version of another computational approach provided in Refs. [17, 41, 42]. It assumes adiabatic separation of the LAM from the other vibrational modes, that we will further refer to as smallamplitude motions (SAM). The latter are to be treated in the harmonic approximation. Our procedure requires two types 
Fig. 1 Structures and proton transfer coordinates of malonaldehyde (MA) and formic acid dimer (FAD). The atomic color schemes are the same for MA and FAD

\section{Malonaldehyde (MA)}

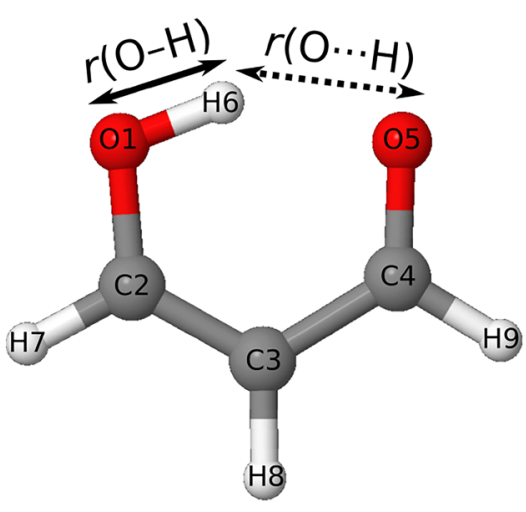

Formic Acid Dimer (FAD)

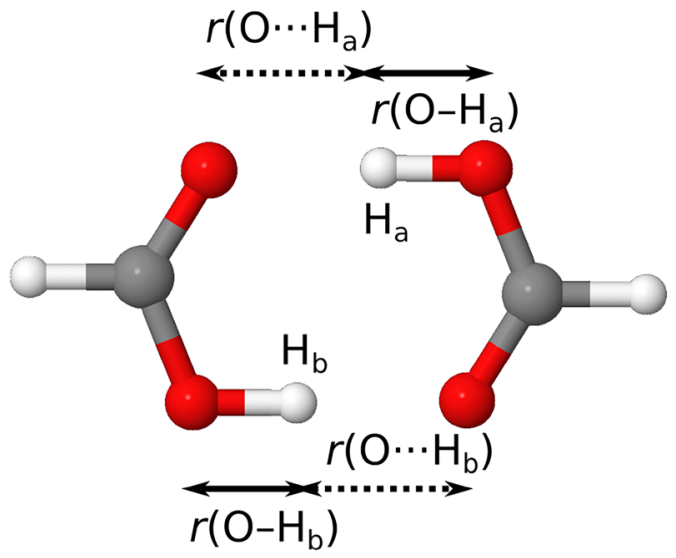

of calculations to be done: one-dimensional (1D) relaxed potential energy surface (PES) scans and vibrational frequency calculations in the harmonic approximation. Both computational procedures are known by users of quantumchemical packages, and thus the presented procedure can become an interesting one for members of the highresolution molecular spectroscopy community. We apply the proposed approach to the nitrogen inversion in ammonia to prove the workability of the method, and then we use it to calculate tunneling splittings for the proton transfer motion in MA and FAD at various quantum-chemical approximations employing the def2-TZVPP basis set.

\section{Computational procedure}

\section{Quantum chemical calculations}

The quantum chemical calculations in the present work were done using Orca 4 software [43]. We have applied the following DFT functionals: PBE [44], PBE0 [45], BLYP, and B3LYP [46-48], CAM-B3LYP [49], B2PLYP [50], and mPW2PLYP [51]. From the post-Hartree-Fock methods, we have applied MP2, SCS-MP2, and all-electron $\operatorname{CCSD}(\mathrm{T})(\mathrm{ae}-\mathrm{CCSD}(\mathrm{T}))$ [52-54]. Some of the calculations were done employing S. Grimme's D3 dispersion correction with Becke - Johnson (BJ) damping [55]. We have applied the resolution-of-identity (RI) technique in order to reduce the computational efforts: we have applied the RIJCOSX method for MA, FAD and B3LYP-D3BJ and CAM-B3LYP calculations of ammonia, while for MP2 and B2PLYP calculations of ammonia, we have used the RI-JK method [56-62]. Basis sets of our choice were def2-nVP, def2-nVPD $(\mathrm{n}=\mathrm{S}, \mathrm{TZ}, \mathrm{QZ})$, and def2-TZVPP, as well as their corresponding auxiliary basis sets [63-66].

\section{Theoretical framework}

The treatment of the LAM that we implemented resembles the procedure introduced in Refs. [41, 42]. It is a mixture of the RSH approach $[1,28,67-70]$, with the kinetic energy expression taken from the Meyer-Günthard Hamiltonian [71]. The Hamiltonian is expressed as

$\hat{H}=-\frac{1}{2} \hat{p}_{\xi} G(\xi) \hat{p}_{\xi}+V(\xi)+\hat{H}_{\mathrm{b}}(\xi)$,

where $\xi$ is the LAM coordinate, $\hat{p}_{\xi}=-i \hbar \partial / \partial \xi=-i \hbar \partial_{\xi}$ is the momentum conjugated to $\xi, V$ is the electronic potential energy surface (PES) along the LAM coordinate (obtained from a relaxed PES scan), and $\hat{H}_{\mathrm{b}}$ is the bath of all other $3 N-7$ SAM degrees of freedom ( $N$ denotes the number of atoms in the molecule). $G=\mu^{-1}$ is the inverted reduced mass $(\mu)$ of the LAM. It is being calculated from the generalized tensor of inertia defined as

$\mathcal{G}^{-1}=\left(\begin{array}{ll}\mathcal{I}_{\mathrm{rr}} & \mathbf{I}_{\mathrm{rv}} \\ \mathbf{I}_{\mathrm{rv}}^{\mathrm{I}} & I_{\mathrm{vv}}\end{array}\right)$

where $\mathcal{I}_{\text {rr }}=\sum_{k=1}^{N} m_{k}\left(\operatorname{diag}\left(\mathbf{r}_{k} \cdot \mathbf{r}_{k}\right)-\mathbf{r}_{k} \otimes \mathbf{r}_{k}\right)$ is the $3 \times 3$ tensor of inertia, $\mathbf{I}_{\mathrm{rv}}=\sum_{k=1}^{N} m_{k}\left[\mathbf{r}_{k} \times \partial_{\xi} \mathbf{r}_{k}\right]$ is the vector of LAM-rotation interaction, and $I_{\mathrm{vv}}=\sum_{k=1}^{N} m_{k}\left(\partial_{\xi} \mathbf{r}_{k} \cdot \partial_{\xi} \mathbf{r}_{k}\right)$. Here, $m$ and $\mathbf{r}$ denote the atomic masses and positions. ${ }^{1} G$ is the last diagonal element of matrix $\mathcal{G}^{-1}$.

Let $\mathbf{r}=\left(x_{1}, y_{1}, z_{1}, x_{2} \ldots\right)^{\dagger}$ be the $3 \mathrm{~N}$-sized vector with Cartesian coordinates of atoms, and $\mathbf{m}=\left(m_{1}, m_{1}, m_{1}, m_{2}, \ldots\right)^{\dagger}$ $3 N$-sized vector of atomic masses. Let $\mathbf{R}=\sqrt{\mathbf{m r}}$ be massweighted coordinates (MWC) of the system, ${ }^{2}$ and $\mathcal{F}(\xi)$ - the Wilson's GF-matrix [72] composed of $\mathcal{F}_{i j}=\partial_{R_{i}} \partial_{R_{j}} V=$

\footnotetext{
$\overline{1 \cdot, \times \text {, and } \otimes}$ denote scalar, cross, and outer products of the vectors. $2 \sqrt{\mathbf{m}}=\left(\sqrt{m_{1}}, \sqrt{m_{2}}, \sqrt{m_{2}}, \sqrt{m_{2}}, \ldots\right)^{\dagger}$.
} 
$\partial_{r_{i}} \partial_{r_{j}} V / \sqrt{m_{i} m_{j}}$ elements. $\mathbf{R}_{\mathrm{eq}}(\xi)=\sqrt{\mathbf{m}} \mathbf{r}_{\mathrm{eq}}(\xi)$ denotes the equilibrium values of atomic coordinates at the LAM coordinate value $\xi$. Then the harmonic vibrational Hamiltonian $\hat{H}_{\mathrm{b}}$ is given as [73]:

$\hat{H}_{\mathrm{b}}(\xi)=\frac{1}{2} \hat{\mathbf{P}}_{\mathrm{MWC}}^{2}+\frac{1}{2}\left(\mathbf{R}-\mathbf{R}_{\mathrm{eq}}(\xi)\right)^{\dagger} \mathcal{F}(\xi)\left(\mathbf{R}-\mathbf{R}_{\mathrm{eq}}(\xi)\right)$,

where $\hat{\mathbf{P}}_{\mathrm{MWC}}=-i \hbar \partial_{\mathbf{R}}$.

We can remove translations/rotations of the molecule as a whole, as well as the LAM, from $\mathcal{F}(\xi)$ using a projector matrix $\mathcal{S}(\xi)$, which can be constructed e.g. using the Gram-Schmidt process. As a result, one can get the harmonic bath GF-matrix $\mathcal{F}_{3 N-7}(\xi)=\mathcal{S}^{\dagger}(\xi) \mathcal{F}(\xi) \mathcal{S}(\xi)$. We chose one of the structures (at $\xi=\xi_{\text {ref }}$ ) as the reference. In our double-well cases, we chose the transition state with $\xi_{\text {ref }}=0$. Then we define the vibrational state of the bath using the normal modes of the reference structure $\left(\mathcal{L}_{\text {ref }}\right)$ given as diagonalizing matrix for $\mathcal{F}_{3 N-7}\left(\xi_{\text {ref }}\right)$ $\left(\mathcal{L}_{\text {ref }}^{\dagger} \mathcal{F}_{3 N-7}\left(\xi_{\text {ref }}\right) \mathcal{L}_{\text {ref }}=\operatorname{diag}\left(\boldsymbol{\Omega}_{\text {ref }}^{2}\right)\right.$, with $\boldsymbol{\Omega}_{\text {ref }}^{2}$ being a vector of squared angular frequencies) $[72,73]$. This allows us to write $\left(\mathbf{R}-\mathbf{R}_{\mathrm{eq}}(\xi)\right)=\mathcal{L}_{\text {ref }} \mathbf{Q}$, where $\mathbf{Q}$ is the position of the nuclei along the normal modes expressed in mass-weighted coordinates. Note that this assumption requires all the structures to be oriented with respect to the reference coordinates. These operations give the harmonic bath Hamiltonian in the form

$$
\begin{aligned}
\hat{H}_{\mathrm{b}}(\xi)= & \underbrace{\frac{1}{2} \sum_{k}\left(\hat{P}_{k}^{2}+\omega_{k}^{2} \hat{Q}_{k}^{2}\right)}_{\hat{H}_{0}}+\underbrace{\frac{1}{2} \sum_{k}\left(\Omega_{k k}^{2}-\omega_{k}^{2}\right) \hat{Q}_{k}^{2}}_{\hat{W}_{1}} \\
& +\underbrace{\frac{1}{2} \sum_{k} \sum_{l \neq k} \Omega_{k l}^{2} \hat{Q}_{k} \hat{Q}_{l}}_{\hat{W}_{2}},
\end{aligned}
$$

where $k$ enumerates normal modes, $\hat{P}_{k}=-i \hbar \partial_{Q_{k}}, \Omega_{k l}^{2}$ are the elements of the matrix $\boldsymbol{\Omega}^{2}(\xi)=\mathcal{L}_{\text {ref }}^{\dagger} \mathcal{F}_{3 N-7}(\xi) \mathcal{L}_{\text {ref }}$, and $\omega_{k}$ are the zeroth approximation frequencies. We can either set them at $\boldsymbol{\Omega}_{\text {ref }}$ values, or allow bath vibrations to relax during the LAM by setting $\omega_{k}$ to the respective diagonal values of $\boldsymbol{\Omega}^{2}(\xi) . \hat{H}_{0}$ denotes the unperturbed vibrational Hamiltonian, and the $\hat{W}_{k}$ are the perturbation operators.

The mass-weighted $\hat{P}_{k} / \hat{Q}_{k}$ (normal modes momentum/ position operators) are related to dimension-less operators $\hat{p}_{k}, \hat{q}_{k}$ corresponding to an oscillator with reference frequency $\omega_{k}$ as

$\left\{\begin{array}{l}\hat{p}_{k}=\frac{\hat{P}_{k}}{\sqrt{\hbar \omega_{k}}}, \\ \hat{q}_{k}=\sqrt{\frac{\omega_{k}}{\hbar}} \hat{Q}_{k} .\end{array}\right.$

We can use them to rewrite the Eq. 3 as

$$
\begin{aligned}
\hat{H}_{\mathrm{b}}(\xi)= & \underbrace{\sum_{k} \hbar \omega_{k}\left(\hat{n}+\frac{1}{2}\right)}_{\hat{H}_{0}}+\underbrace{\sum_{k} \frac{\hbar}{2 \omega_{k}}\left(\Omega_{k k}^{2}-\omega_{k}^{2}\right) \hat{q}_{k}^{2}}_{\hat{W}_{1}} \\
& +\underbrace{\sum_{k} \sum_{l \neq k} \frac{\hbar \Omega_{k l}^{2}}{2 \sqrt{\omega_{k} \omega_{l}}} \hat{q}_{k} \hat{q}_{l}}_{\hat{W}_{2}},
\end{aligned}
$$

where $\hat{n}=\hat{a}^{\dagger} \hat{a}$ with $\hat{a}=\frac{\hat{q}_{k}+i \hat{p}_{k}}{\sqrt{2}}$ and $\hat{a}^{\dagger}=\frac{\hat{q}_{k}-i \hat{p}_{k}}{\sqrt{2}}$ (annihilation/ creation operators). ${ }^{3}$

If we consider the bath to be in a chosen vibrational eigenstate $|\mathbf{v}\rangle=\prod_{k}\left|v_{k}\right\rangle$ (with $\mathbf{v}=\left(v_{1}, v_{2}, \ldots\right)$ being a vector of quantum numbers $\left.v_{k}=0,1,2, \ldots\right)$ of the Hamiltonian $\hat{H}_{0}$ $\left(\hat{H}_{0}|\mathbf{v}\rangle=\sum_{k} \hbar \omega_{k}\left(v_{k}+1 / 2\right)|\mathbf{v}\rangle\right)$, then we can apply perturbation theory (PT) to get the expression of the bath energy. Using the second quantization form of $\hat{p}_{k}$ and $\hat{q}_{k}$, one can compute the bath Hamiltonian energies.

$-0^{\text {th }}$ order.

$$
E_{\mathbf{v}}^{(0)}=\left\langle\mathbf{v}\left|\hat{H}_{0}\right| \mathbf{v}\right\rangle=\sum_{k} \hbar \omega_{k}\left(v_{k}+\frac{1}{2}\right),
$$

$-1^{\text {st }}$ order correction $\left(\left\langle\mathbf{v}\left|\hat{W}_{2}\right| \mathbf{v}\right\rangle=0\right) .^{4}$

$$
\delta E_{\mathbf{v}}^{(1)}=\left\langle\mathbf{v}\left|\hat{W}_{1}\right| \mathbf{v}\right\rangle=\sum_{k} \frac{\hbar}{2 \omega_{k}}\left(\Omega_{k k}^{2}-\omega_{k}^{2}\right)\left(v_{k}+\frac{1}{2}\right) .
$$

$-2^{\text {st }}$ order correction:

$$
\begin{aligned}
\delta E_{\mathbf{v}}^{(2.1)} & =-\sum_{\mathbf{v}^{\prime} \neq \mathbf{v}} \frac{\left|\left\langle\mathbf{v}^{\prime}\left|\hat{W}_{1}\right| \mathbf{v}\right\rangle\right|^{2}}{E_{\mathbf{v}^{\prime}}^{(0)}-E_{\mathbf{v}}^{(0)}} \\
& =-\sum_{k} \frac{\hbar\left(\Omega_{k k}^{2}-\omega_{k}^{2}\right)^{2}}{8 \omega_{k}^{3}}\left(v_{k}+\frac{1}{2}\right),
\end{aligned}
$$

and

$$
\begin{aligned}
\delta E_{\mathbf{v}}^{(2.2)} & =-\sum_{\mathbf{v}^{\prime} \neq \mathbf{v}} \frac{\left|\left\langle\mathbf{v}^{\prime}\left|\hat{W}_{2}\right| \mathbf{v}\right\rangle\right|^{2}}{E_{\mathbf{v}^{\prime}}^{(0)}-E_{\mathbf{v}}^{(0)}} \\
& =-\sum_{k} \sum_{l \neq k} \frac{\hbar \Omega_{k l}^{4}}{4 \omega_{k} \omega_{l}} \frac{\omega_{k}\left(v_{l}+\frac{1}{2}\right)-\omega_{l}\left(v_{k}+\frac{1}{2}\right)}{\omega_{k}^{2}-\omega_{l}^{2}}
\end{aligned}
$$

The resulting energy is:

\footnotetext{
$\overline{3}$ I.e. $\hat{a}|v\rangle=\sqrt{v}|v-1\rangle, \hat{a}^{\dagger}|v\rangle=\sqrt{v+1}|v+1\rangle$ and $\hat{n}|v\rangle=v|v\rangle$.

${ }^{4}$ Using the fact that $\hat{q}^{2}=\frac{\left(\hat{a}^{\dagger}\right)^{2}+\hat{a}^{2}}{2}+\hat{n}+\frac{1}{2}$.
} 


$$
\begin{aligned}
E_{\mathbf{v}} \approx & \underbrace{\sum_{k} \hbar \frac{3 \omega_{k}^{4}+6 \Omega_{k k}^{2} \omega_{k}^{2}-\Omega_{k k}^{4}}{8 \omega_{k}^{3}}\left(v_{k}+\frac{1}{2}\right)}_{E_{\mathbf{v}}^{(0)}+\delta E_{\mathbf{v}}^{(1)}+\delta E_{\mathbf{v}}^{(2.1)}} \\
& -\sum_{\delta E_{\mathbf{v}}^{(2.2)}} \sum_{l \neq k} \frac{\hbar \Omega_{k l}^{4}}{4 \omega_{k} \omega_{l}} \frac{\omega_{k}\left(v_{l}+\frac{1}{2}\right)-\omega_{l}\left(v_{k}+\frac{1}{2}\right)}{\omega_{k}^{2}-\omega_{l}^{2}}
\end{aligned}
$$

For the pure LAM excitations we can assume that the other molecular degrees of freedom are at the ground state $(\mathbf{v}=\mathbf{0})$, and therefore, the energy of the bath is the zeropoint vibrational energy (ZPVE) given by an expression

$\mathrm{ZPVE}=\sum_{k} \hbar \frac{3 \omega_{k}^{4}+6 \Omega_{k k}^{2} \omega_{k}^{2}-\Omega_{k k}^{4}}{16 \omega_{k}^{3}}-\sum_{k} \sum_{l \neq k} \frac{\hbar \Omega_{k l}^{4}}{8 \omega_{k} \omega_{l}\left(\omega_{k}+\omega_{l}\right)}$.

If we allow vibrational states relaxation, then $\omega_{k}=\Omega_{k k}$, which gives

$\operatorname{ZPVE}(\xi)=\sum_{k} \frac{\hbar \Omega_{k k}(\xi)}{2}-\sum_{k} \sum_{l \neq k} \frac{\hbar \Omega_{k l}^{4}(\xi)}{8 \Omega_{k k}(\xi) \Omega_{l l}(\xi)\left(\Omega_{k k}(\xi)+\Omega_{l l}(\xi)\right)}$

By combining Eqs. 1 and 6 the final effective Hamiltonian for LAM looks as the following:

$\hat{H}=-\frac{1}{2} \hat{p}_{\xi} G(\xi) \hat{p}_{\xi}+\overbrace{V(\xi)+\operatorname{ZPVE}(\xi)}^{V_{\text {eff }}(\xi)}$.

The parameters for this Hamiltonian (effective masses $G(\xi)$ and effective PES $\left.V_{\text {eff }}(\xi)\right)$ in this work were computed from relaxed PES scans obtained with Orca 4 using in-house Python scripts [74]. In brief, the script for computing the Hamiltonian parameters works as following.

- The code reads Orca output * . hess files containing vibrational information (molecular geometry, atomic masses, Hessian, etc.).

- The molecular geometries and Hessians are oriented with respect to the reference geometry defined as the molecular structure with the lowest value of $|\xi|$ (the closest to the transition state). The orientation corresponds to the minimum of the functional $\sum_{k=1}^{N} m_{k}\left(\mathcal{R} \mathbf{r}_{k}(\xi)-\mathrm{r}_{\text {ref }, k}\right)^{2}$, where $\mathcal{R}$ is the rotation matrix.

- The effective masses $\mu=G^{-1}$ are computed as described above. The derivatives $\frac{d \mathbf{r}_{k}}{d \xi}(\xi)$ are computed by fitting the expression $\mathbf{r}_{k}\left(\xi^{\prime}\right) \approx \mathbf{r}_{k}(\xi)+\frac{d \mathbf{r}_{k}}{d \xi}(\xi)\left(\xi^{\prime}-\xi\right)$ to the nearest structures with linear least-squares method.

- The vibrational problem for each structure is solved with exclusion of translations, rotation and LAM direction, given by the $\frac{d \mathbf{r}_{k}}{d \xi}(\xi)$ vector, the spurious imaginary fre- quencies are also being set to zero. The resulting Hessians are stored.

- The ZPVE correction is computed for each structure by projecting the reference structure normal modes onto the Hessians retrieved in the previous item.

- The obtained dependencies for $\mu(\xi), V(x)$ and ZPVE $(\xi)$, are extrapolated using cubic splines to a fine uniform grid.

The resulting eigenvalue problem is solved using the discrete variable representation (DVR) method, in particular, sincDVR [75].

\section{Results and discussion}

\section{Proof of concept: ammonia}

To test the performance of the implementation, we chose the simplest case of LAM with tunneling: an inversion motion in ammonia $\left(\mathrm{NH}_{3}\right)$. The LAM coordinate was defined as

$\xi_{\mathrm{NH}_{3}}=\frac{\mathbf{r}_{\mathrm{NH} 1} \cdot\left[\mathbf{r}_{\mathrm{NH} 2} \times \mathbf{r}_{\mathrm{NH} 3}\right]}{2 \cdot \sqrt{s \cdot\left(s-r_{12}\right) \cdot\left(s-r_{13}\right) \cdot\left(s-r_{23}\right)}}$,

where $\mathbf{r}_{\mathrm{NH} n}$ denotes a position vector of the hydrogen number $n$ with nitrogen as an origin, $r_{n m}=\left|\mathbf{r}_{\mathrm{NH} n}-\mathbf{r}_{\mathrm{NH} m}\right|$, and $s=\left(r_{12}+r_{13}+r_{23}\right) / 2$. The $\xi_{\mathrm{NH}_{3}}$ is basically a height $(h)$ of the tetrahedron constructed on the $\mathbf{r}_{\mathrm{NH} n}$ vectors with an origin at the nitrogen atom, computed through the volume of the tetrahedron $V=A \cdot h / 3$ and the area of the base of the tetrahedron $(A)$.

The 1D PES scans were performed at B3LYP-D3BJ, CAM-B3LYP, MP2, and B2PLYP levels of theory. The resulting parameters of the LAM Hamiltonian (Eq. 7) are given in Fig. 2. All the methods performed similar to each other. The energy levels of ammonia and some of its isotopologues obtained with CAM-B3LYP are given in Table 1. Concerning simplicity of the calculation, the results are very similar to the experimental values. The values of other methods can be found in the SI. The results for ammonia confirm the workability of the code, which justify testing of the approach on larger molecules.

\section{Proton transfer in malonaldehyde (MA) and formic acid dimer (FAD)}

\section{Tunneling splittings in the parent species}

The PESs for proton transfer in MA and FAD were computed at various levels of theory, using both post-Hartree Fock methods (MP2 and SCS-MP2) and different DFT functionals 

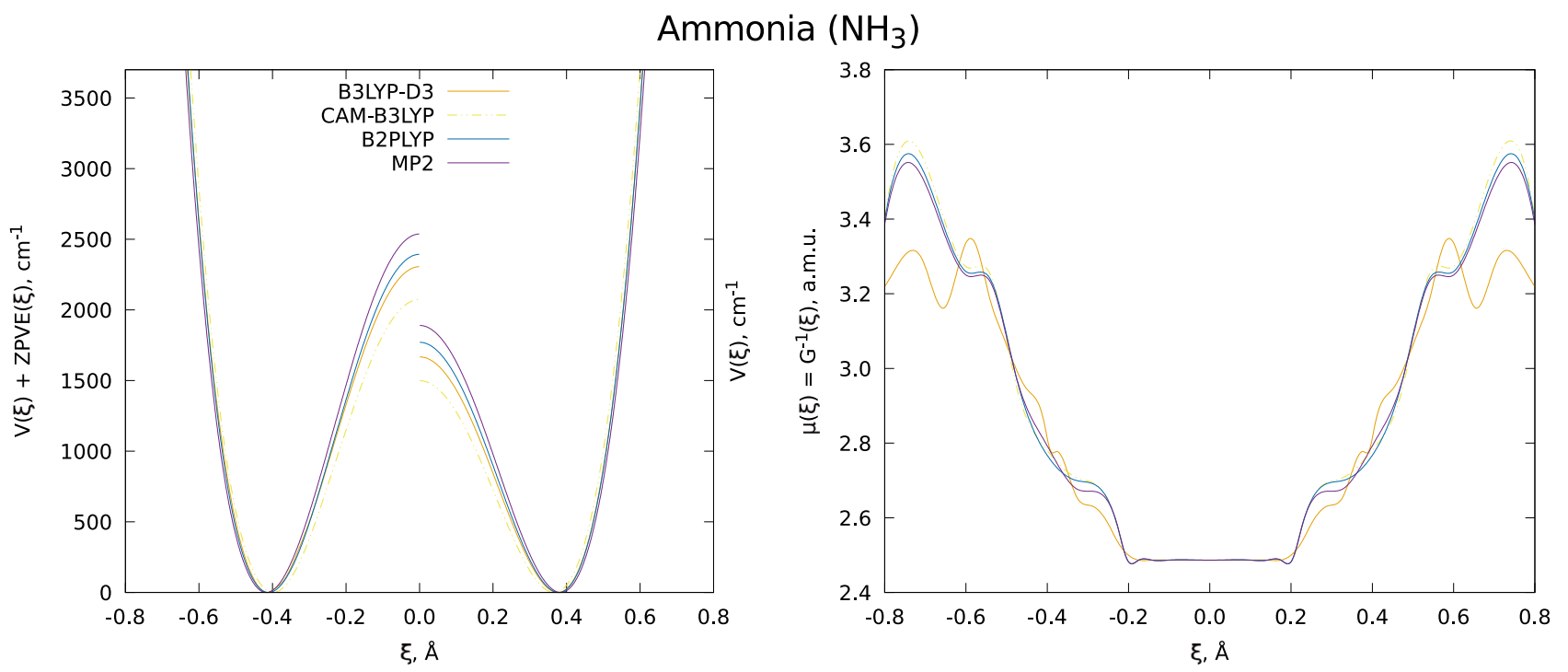

Fig. 2 Effective $(V(\xi)+\mathrm{ZPVE}(\xi))$ and normal $(V(\xi))$ potential energy surfaces, and effective masses $\mu(\xi)$ of inversion for the parent isotopologue of ammonia computed at various levels of theory with def2-TZVPP basis set. The reaction coordinate $\xi$ is given by Eq. 8

from different steps of Perdew's ladder [76]: GGA (PBE and BLYP), hybrid (B3LYP and PBE0), range-separated hybrid (CAM-B3LYP), and double hybrid (B2PLYP and mPW2PLYP). FAD is a dimer; therefore, we took into account possible missing dispersion interactions in case of GGA and hybrid functionals via D3BJ correction. The same correction was also tested together with one of the double-hybrid functionals (B2PLYP).

The proton transfer coordinates were as following. In case of MA:

$\xi=\frac{r(\mathrm{O} \cdots \mathrm{H})-r(\mathrm{O}-\mathrm{H})}{2}$,

and for FAD as

$\xi=\frac{r\left(\mathrm{O}-\mathrm{H}_{a}\right)+r\left(\mathrm{O}-\mathrm{H}_{b}\right)-r\left(\mathrm{O} \cdots \mathrm{H}_{\mathrm{a}}\right)-r\left(\mathrm{O} \cdots \mathrm{H}_{\mathrm{b}}\right)}{2 \sqrt{2}}$.
The obtained Hamiltonian parameters (PES and effective masses) for these calculations are plotted in Fig. 3, the computed tunneling splittings between ground vibrational states of different parity $\left(0^{+} / 0^{-}\right)$are given in Table 2 . As it can be seen from these results, most of the tested methods give reasonable estimates of the tunneling splittings compared to the experimental values determined in Refs. $[6,9,15]$. The pure GGA functionals with dispersion corrections (PBED3BJ and BLYP-D3BJ), as well as the hybrid PBE0-D3BJ, perform not well due to underestimation of the proton transfer barriers. Double hybrids (especially B2PLYP without D3BJ), MP2, and CAM-B3LYP work stable for both MA and FAD. B3LYP-D3BJ performs well for MA, but in case of FAD, it underestimates the barrier height, which might be a result of the intramolecular nature of the bonding forces in this complex. SCS-MP2 showed the worst performance.
Table 1 Vibrational state energies for the inversion of ammonia and some of its isotopologues. Experimental values are taken from Ref. [12], theoretical values are computed with a scan obtained at the CAM-B3LYP/def2-TZVPP level of theory. All the values are in $\mathrm{cm}^{-1}$

\begin{tabular}{|c|c|c|c|c|c|c|c|c|}
\hline \multirow[t]{2}{*}{ State } & \multicolumn{2}{|l|}{$\mathrm{NH}_{3}$} & \multicolumn{2}{|l|}{$\mathrm{ND}_{3}$} & \multicolumn{2}{|l|}{${ }^{15} \mathrm{ND}_{3}$} & \multicolumn{2}{|l|}{$\mathrm{NT}_{3}$} \\
\hline & Exp. & Theor. & Exp. & Theor. & Exp. & Theor. & Exp. & Theor. \\
\hline $0^{+}$ & 0 & 0 & 0 & 0 & 0 & 0 & 0 & 0 \\
\hline $0^{-}$ & 0.76 & 0.59 & 0.05 & 0.06 & 0.05 & 0.05 & 0.01 & 0.01 \\
\hline $1^{+}$ & 928.5 & 948.1 & 745.6 & 740.7 & 739.5 & 734.7 & 656.4 & 647.4 \\
\hline $1^{-}$ & 962.9 & 978.4 & 749.2 & 744.9 & 742.8 & 738.5 & 656.4 & 648.6 \\
\hline $2^{+}$ & 1591.2 & 1623.8 & 1359.0 & 1337.3 & & & & \\
\hline $2^{-}$ & 1870.9 & 1889.0 & 1429.0 & 1416.8 & & & & \\
\hline $3^{+}$ & 2369.3 & 2396.0 & 1830.0 & 1807.2 & & & & \\
\hline $3^{-}$ & 2876.1 & 2909.6 & 2106.6 & 2101.3 & & & & \\
\hline
\end{tabular}


Malonaldehyde (MA)
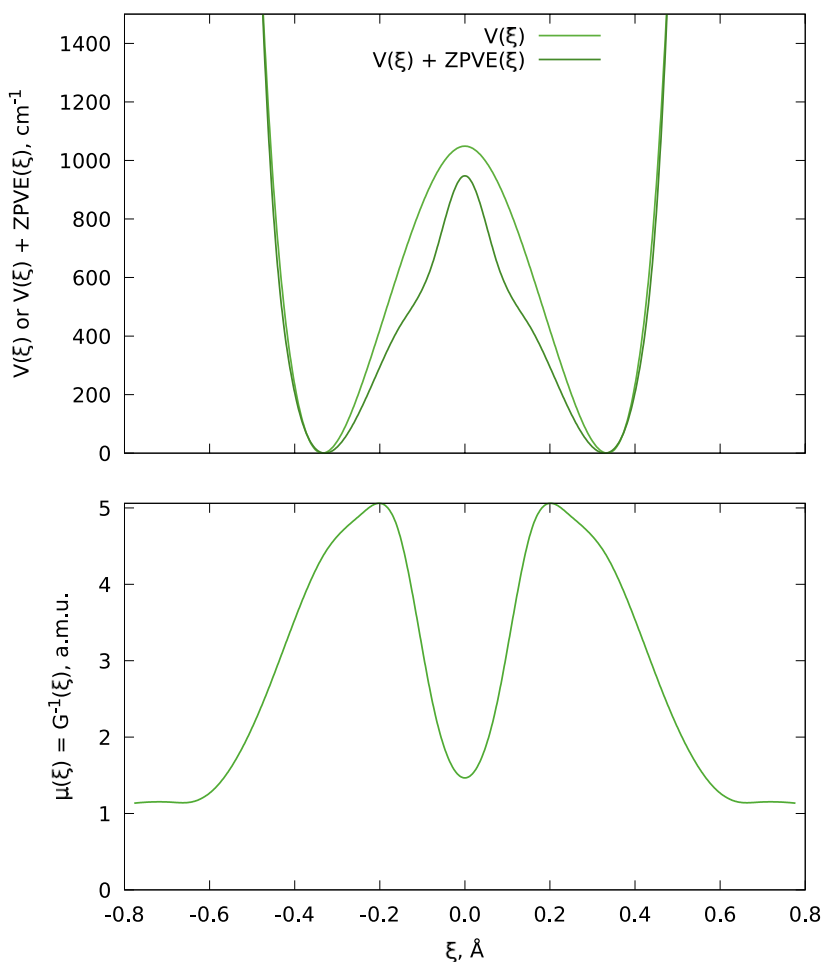

Fig. 3 Effective $(V(\xi)+\mathrm{ZPVE}(\xi))$ and normal $(V(\xi))$ potential energy surfaces and effective masses $\mu(\xi)$ of proton transfer for the parent isotopologues of MA and FAD computed at B2PLYP/def2-

\section{Tunneling splittings in the MA isotopologues}

Similar to ammonia, MA has experimental information available on the tunneling splittings in its isotopologues [7, 8]. These parameters were calculated for MA-D8, MA-D6 and MA-D6D8 isotopologues at B3LYP-D3BJ, CAM-B3LYP, B2PLYP and MP2 levels of theory, with def2-TZVPP basis
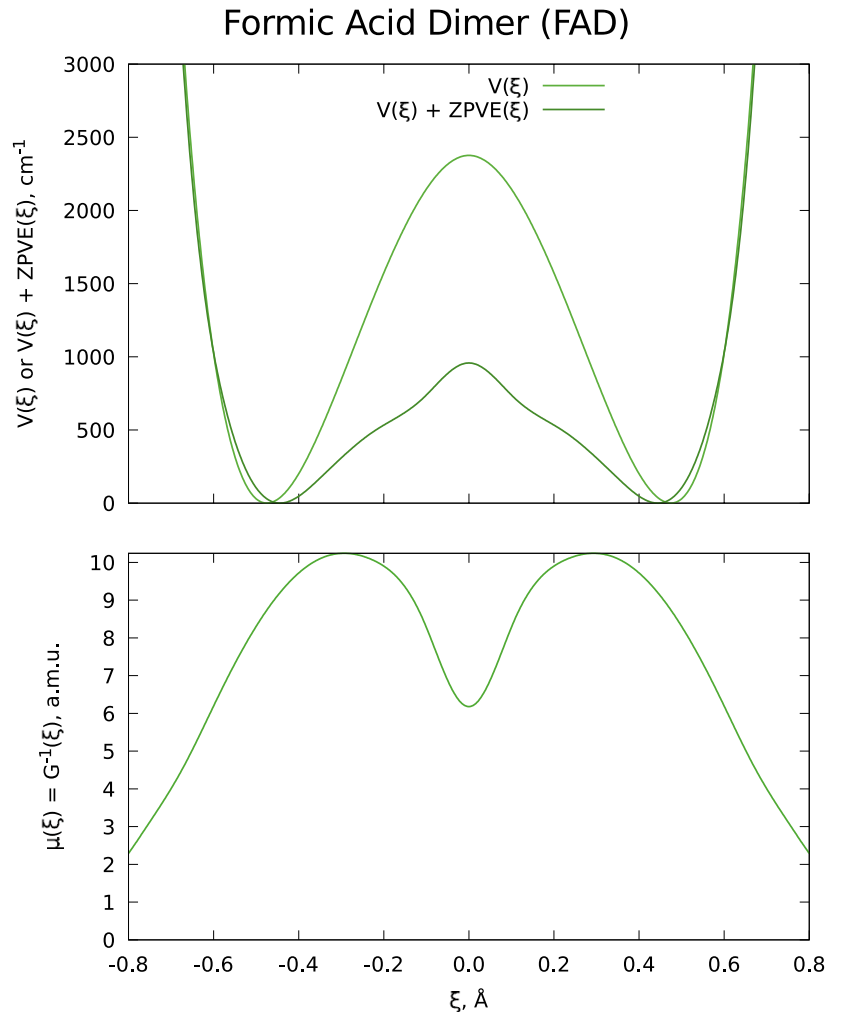

TZVPP level of theory. The reaction coordinates $\xi$ for MA and FAD are given by Eqs. 9 and 10, respectively. Data sets for the other tested levels of theory are available in SI

set. The results are given in Table 3. The value for the MA-D8 conformer is close to the parent isotopologue. This is expected, since the hydrogen number 8 is on the opposite side of the molecule with respect to the proton transfer $\mathrm{O}-\mathrm{H} \cdots \mathrm{O}$ fragment. The MA-D6 and MA-D6D8 isotopologues, as expected, show lower tunneling splitting values, since hydrogen number 6 is the one being transferred between the oxygens during the
Table $20^{+} / 0^{-}$tunneling splittings $\left(\Delta E_{ \pm}\right)$, reaction barriers $\left(E_{\mathrm{BH}}\right)$, and effective reaction barriers $\left(E_{\mathrm{BH}}+\mathrm{ZPVE}\right)$ for proton transfer motions in MA and FAD obtained with def2-TZVPP basis sets. Experimental values were taken from Ref. [6] for MA and from Refs. $[9,15]$ for FAD. All the values are in $\mathrm{cm}^{-1}$

\begin{tabular}{|c|c|c|c|c|c|c|}
\hline \multirow[t]{2}{*}{ Method } & \multicolumn{3}{|l|}{ MA } & \multicolumn{3}{|l|}{ FAD } \\
\hline & $\Delta E_{ \pm}$ & $E_{\mathrm{BH}}$ & $E_{\mathrm{BH}}+\mathrm{ZPVE}$ & $\Delta E_{ \pm}$ & $E_{\mathrm{BH}}$ & $E_{\mathrm{BH}}+\mathrm{ZPVE}$ \\
\hline PBE-D3BJ & 89.0 & 342 & 443 & 1.591 & 1107 & 655 \\
\hline BLYP-D3BJ & 59.0 & 743 & 457 & 2.370 & 1764 & 463 \\
\hline PBE0-D3BJ & 51.1 & 747 & 652 & 3.989 & 1716 & 380 \\
\hline B3LYP-D3BJ & 17.4 & 1095 & 974 & 0.115 & 2205 & 703 \\
\hline CAM-B3LYP & 19.7 & 1078 & 966 & 0.073 & 2139 & 722 \\
\hline B2PLYP & 20.3 & 1049 & 947 & 0.019 & 2376 & 958 \\
\hline B2PLYP-D3BJ & 17.2 & 1059 & 972 & 0.035 & 2353 & 910 \\
\hline mPW2PLYP & 14.8 & 1142 & 1050 & 0.014 & 2450 & 982 \\
\hline MP2 & 28.8 & 953 & 897 & 0.059 & 2235 & 899 \\
\hline SCS-MP2 & 1.5 & 1544 & 1605 & $1 \cdot 10^{-4}$ & 2951 & 1510 \\
\hline Experiment & $21.5831383(6)$ & & & $0.01117 / 0.011367(92)$ & & \\
\hline
\end{tabular}


Table 3 Tunneling splittings for proton transfer in MA and some of its isotopologues. See Fig. 1 and Refs. [7, 8] for enumeration of the atoms. All the values are in $\mathrm{cm}^{-1}$

\begin{tabular}{llllll}
\hline Isotopologue & Exp. & B3LYP-D3BJ & CAM-B3LYP & B2PLYP & MP2 \\
\hline MA-parent & $21.5831383(6)$ & 17.3 & 19.6 & 20.3 & 28.8 \\
MA-D8 & 26(10) & 17.0 & 19.4 & 20.0 & 28.4 \\
MA-D6 & $2.915(4)$ & 9.2 & 7.8 & 7.2 & 10.8 \\
MA-D6D8 & $2.88360(2)$ & 9.0 & 7.6 & 7.1 & 10.6 \\
\hline
\end{tabular}

LAM. However, the agreement with experimental values is worse compared to the parent MA. This is not observed in the case of $\mathrm{NH}_{3}$, where all the vibrational levels for the substituted isotopologues are predicted with approximately the same level of accuracy as for the parent species.

The most probable explanation to that is breaking of the adiabaticity assumed in the Hamiltonian given in Eq. 2. Isotopic substitution that leads to an increase of the atomic masses with respect to the parent isotopologue (deuteration, ${ }^{13} \mathrm{C}$ and ${ }^{15} \mathrm{~N}$ substitution) reduces the vibrational frequencies of the SAM in the bath. This can make these SAM susceptible to
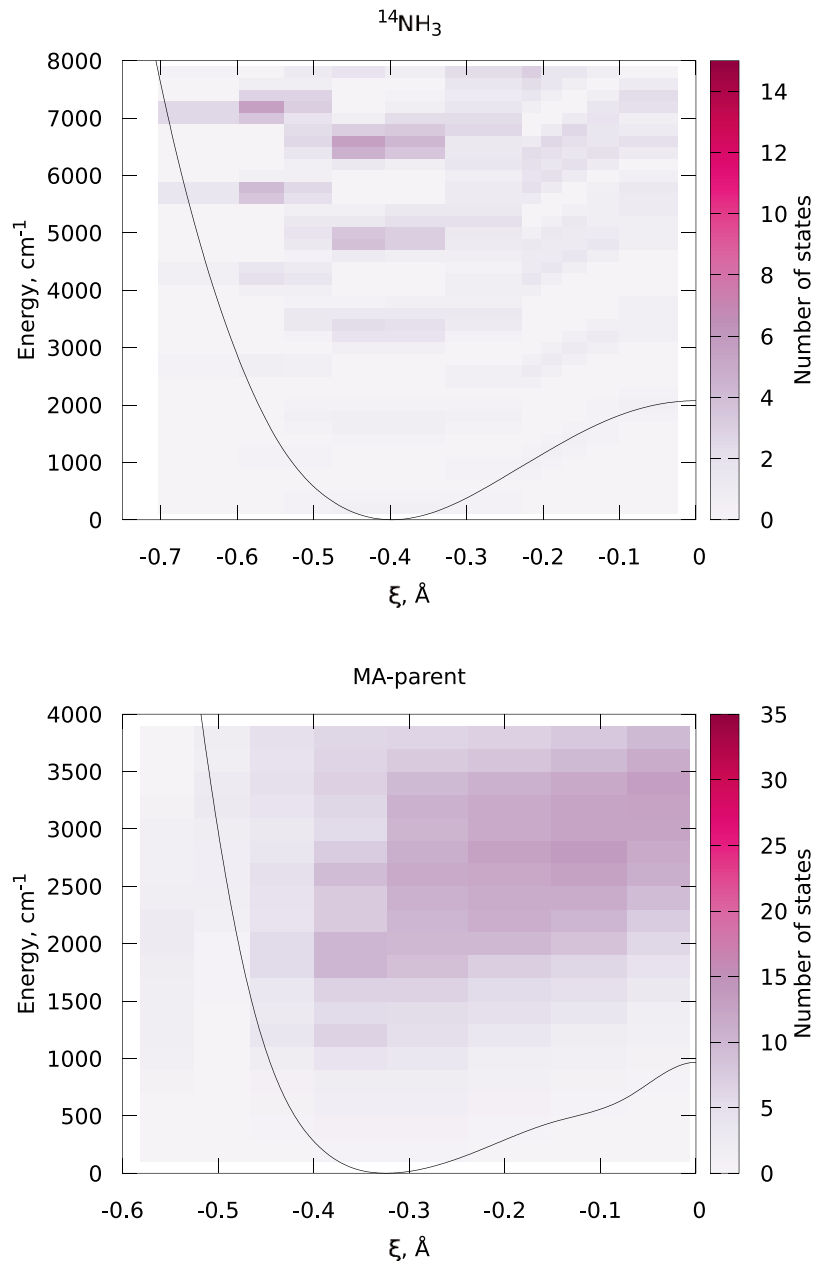

Fig. 4 Number of vibrational states $V_{\mathbf{v}}(\xi)=V(\xi)+E_{\mathbf{v}}(\xi)$ with vibrational energy of the SAM defined by Eq. 5. The black line shows the effective potential $V_{0}(\xi)=V(\xi)+\operatorname{ZPVE}(\xi)$ (Eq. 6) for ammonia vibrational excitation by LAM because of the reduced energy difference between effective potential surfaces. MA has lowenergy vibrational modes, which might lead to this adiabaticity violation. This is supported by the plots of the density of the LAM vibrational manifolds given as $V_{\mathbf{v}}(\xi)=V(\xi)+E_{\mathbf{v}}(\xi)$ with vibrational energy of the SAM defined by Eq. 5 (see Fig. 4). In case of $\mathrm{NH}_{3}, \mathrm{ND}_{3}$, and the parent $\mathrm{MA}$ isotopologue, the effective potential energy $V_{0}(\xi)=V(\xi)+\operatorname{ZPVE}(\xi)$ is well separated from the excited vibrational manifolds. However, in the case of MA-D6, the other manifolds get close to the $V_{0}(\xi)$ at the positions $\xi$, which are populated in the ground vibrational
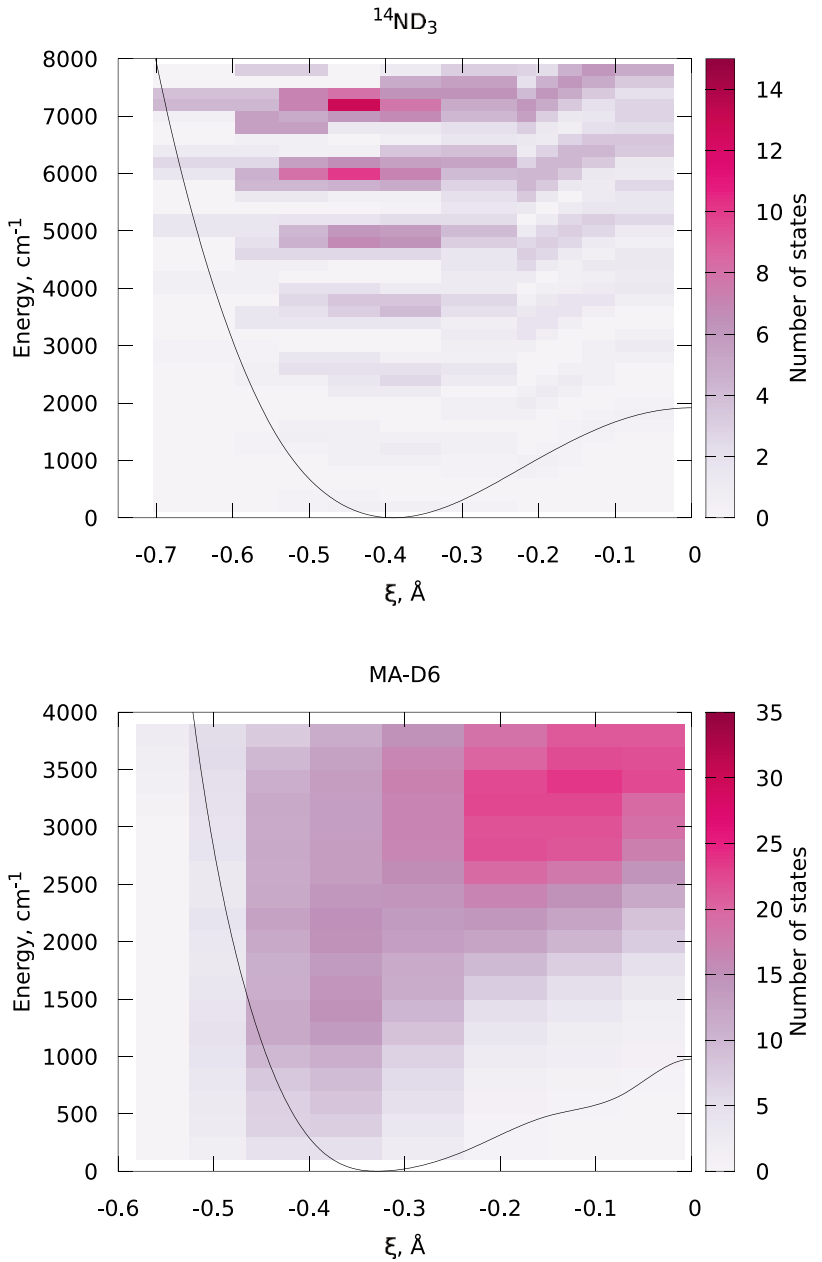

$\left(\mathrm{NH}_{3}\right)$, its fully deuterated isotopologue $\left(\mathrm{ND}_{3}\right)$, MA, and MA-D6 isotopologue (see Fig. 1 for enumeration). Reaction coordinates $\xi$ for ammonia and MA are given by Eqs. 8 and 9, respectively 


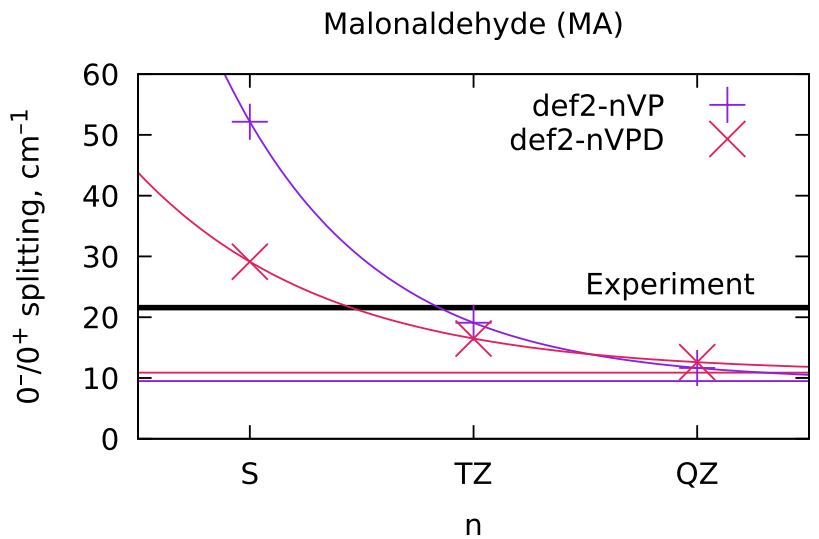

Fig. $50^{+} / 0^{-}$tunneling splittings for proton transfer motion in MA and FAD molecules at B3LYP-D3BJ/def2-nVP and B3LYP-D3BJ/ def2-nVPD levels of theory, and the complete basis set (CBS) extrap-

state. This means that multiple vibrational manifolds might be needed for consideration in order to get a good description of the LAM ground vibrational state of the MA-D6.

\section{Complete basis set (CBS) extrapolation of the tunneling splittings}

Karlsruhe basis set series [66] can be used for the complete basis set (CBS) extrapolation [77, 78], therefore a general possibility of an extrapolation to the complete basis set (CBS) limit for the tunneling splitting values was investigated. For this purpose, we have computed the $0^{+} / 0^{-}$tunneling splittings in MA and FAD using def2-nVP and def2-nVPD basis set series $(\mathrm{n}=$ $\mathrm{S}, \mathrm{TZ}, \mathrm{QZ}$, which corresponds to cardinal number $\zeta=2,3,4$, respectively) with the B3LYP-D3BJ functional. The tunneling splittings were then approximated by an exponential fitting formula $\Delta E_{0^{+} / 0^{-}}(\zeta)=\Delta E_{0^{+} / 0^{-}}^{\mathrm{CBS}}+A \cdot \exp (-\alpha \cdot \zeta)$, where $\Delta E_{0^{+} / 0^{-}}$ denote the value of the splitting obtained at different basis sets and the CBS-extrapolated value. Due to smaller values of the tunneling splitting of FAD, which give rise to larger numerical instabilities, the $\alpha$ parameters (1.5 for def2-nVP and 1.2 for def2-nVPD) for the CBS extrapolation for FAD were taken from the MA fits. The results are shown in Fig. 5. Both def2$\mathrm{nVP}$ and def2-nVPD basis set series converge to similar values of $\Delta E_{0^{+} / 0^{-}}^{\mathrm{CBS}}$. The used basis series consist only of three basis sets, therefore we could not make a proper estimation of the CBS extrapolation errors in each serie, however, the similar trends for the tunneling splittings are clearly visible. The usage of the $\alpha$ parameter obtained from MA for FAD tunneling splittings indicates a transferability of the trends from one molecular system to another. Thus, we conclude that the CBS extrapolation is also, in principle, possible for such complicated parameters as tunneling splittings.

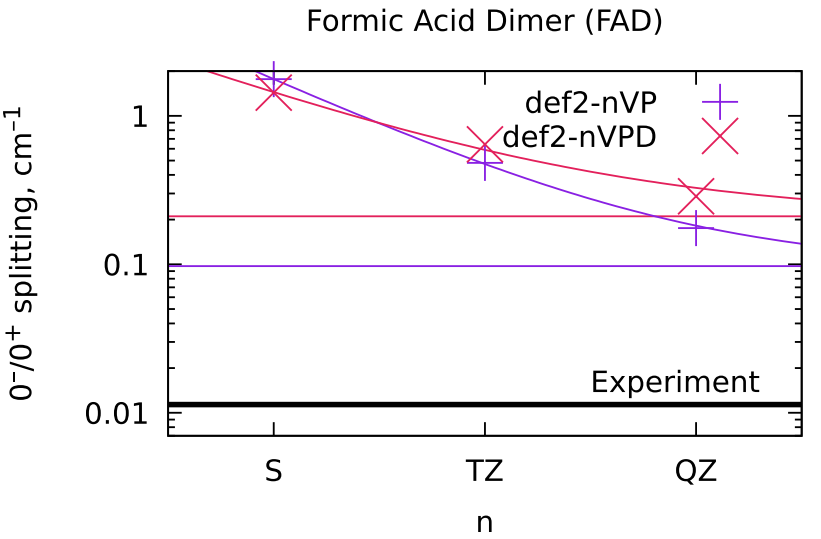

olation of these dependencies (horizontal dashed lines). Solid black horizontal lines denote the experimental values

\section{Reaction coordinate choice invariance}

The proposed approach explicitly relies on the reaction coordinate definition: for calculations of both reduced mass of the motion and of the SAM ZPVE correction. Although, by construction, the Schödinger equation solutions produced by any of the properly chosen coordinates (i.e., by those, that can map a transition from one minimum to another through the vicinity of the transition state) should be the same. However, the numerical implementation of the methods might be rather sensitive to the coordinate choice. In order to check this stability of the algorithm, we have performed the same type of calculation for MA at B2PLYP/def2-TZVPP level of theory with ill-defined reactions coordinates (see Fig. 1 for atomic enumeration):

$\xi_{\mathrm{CH}}=\frac{r(\mathrm{C} 2 \ldots \mathrm{H} 6)-r(\mathrm{C} 4 \ldots \mathrm{H} 6)}{2}$,

and

$\xi_{\mathrm{HH}}=\frac{r(\mathrm{H} 7 \ldots \mathrm{H} 6)-r(\mathrm{H} 9 \ldots \mathrm{H} 6)}{2}$.

The value computed with the initial reaction coordinate (Eq. 9) was $20.3 \mathrm{~cm}-1$ (see Table 2). By replacing the $\xi$ to $\xi_{\mathrm{CH}}$ and $\xi_{\mathrm{HH}}$ the result did not change significantly: 19.9 and $20.1 \mathrm{~cm}-1$, respectively. Thus, we conclude that the implemented method is rather stable with respect to the reaction coordinate definition.

\section{Calculation using the composite approaches}

The additional test of performace was done for the composite quantum chemical calculations $\mathrm{B} / / \mathrm{A}$, where $\mathrm{A}$ 
Table 4 Theoretical $0^{+} / 0^{-}$tunneling splittings for proton transfer motions in MA at different composite methods. The calculations have used the def2-TZVPP basis set. All the values are in $\mathrm{cm}^{-1}$

\begin{tabular}{lc}
\hline Method & Splitting \\
\hline B3LYP-D3BJ & 17.4 \\
MP2 & 28.8 \\
B2PLYP & 20.3 \\
MP2//B3LYP-D3BJ & 21.4 \\
B2PLYP//B3LYP-D3BJ & 17.6 \\
ae-CCSD(T)//B3LYP-D3BJ & 7.0 \\
\hline
\end{tabular}

indicates a computationally cheap quantum chemical method, that is being used as a source of relaxed PES scan and harmonic vibrational frequencies, and $\mathrm{B}$ is the high accuracy level of theory, that provides accurate single point energies. In order to check the possibility of such schemes, we used the relaxed PES scan done at B3LYP-D3BJ/def2-TZVPP level of theory, and computed single point energies at these geometries using the MP2, B2PLYP, and ae-CCSD(T) levels of theory with the def2TZVPP basis set. The computed tunneling splittings are given in Table 4. As one can see, all composite schemes gave as reasonable estimates for the splittings, as the single method calculations. However, the scheme employing the most accurate quantum chemical approximation of all tested (ae-CCSD(T)/def2-TZVPP) gave the splitting 15 wavenumbers lower than the expected value. Such decrease of the value might be caused by a different local shape of the PES at this approximation with respect to the B3LYP-D3BJ/def2-TZVPP. This discrepancy requires additional investigation, which is out of the scope of the current manuscript. Nevertheless, we can conclude that the proposed method for computation of the tunneling splittings should also be applicable with the composite quantum chemical calculations.

\section{Limitations of the approach}

The introduced approach seems to have three main limitation factors for its performance:

- usage of the approximate quantum-chemical methods;

- a few steps in the computational procedure, namely, calculation of the effective masses $(\mu)$, of the ZPVE, and the interpolation, introduce the numerical noise;

- anharmonic effects for the bath vibrational modes.

Despite all three effect, the benchmark calculations given above demonstrate, that with such simple computational procedure we can predict an order of magnitude of the tunneling splitting based only on the quantum-chemical calculations with a relatively low computational cost. Therefore the price-quality ratio of the model given here, seems to be good enough, such as this approach can be used in a routine fashion for augmenting the experimental findings.

\section{Conclusion}

We have introduced, implemented, and tested an approach for computing the tunneling splittings for one-dimensional large amplitude motions (LAMs). The computational scheme is based on the adiabatic separation of LAM from the small amplitude motions, which are treated as a vibrational bath. The bath zero-point vibrational energy (ZPVE) is added to the electronic potential energy surface (PES) to yield an effective PES. Combined with an effective mass, we get a one-dimensional Schrödinger equation, and the solutions of the latter allow for determination of the tunneling splitting caused by the LAM between equivalent minima.

The described approach has been shown to work in cases of three model LAMs: nitrogen inversion in ammonia, single proton transfer in MA, and double proton transfer in FAD. Most of the tested methods (B3LYP-D3BJ, CAM-B3LYP, B2PLYP, mPW2PLYP, B2PLYP-D3BJ, and MP2) performed reasonably well and thus can be recommended for general usage. However, the pure GGA functionals tested (PBE-D3BJ and BLYP-D3BJ), as well as the PBE0-D3BJ and SCS-MP2 methods give splitting values that are quite far away from the experimental values and thus should be avoided in the calculations of the proton transfer tunneling splittings. The methodology with certain limitations was found to be applicable for isotopic substitution: an applicability condition is that the adiabatic separation of the LAM from SAM should remain in the isotopologues. We have also demonstrated a possibility of the CBS extrapolation for the tunneling splittings, the of the usage of the composite quantum chemical methods, and the invariance of the calculated spliting with respect to the reaction coordinate choice. In conclusion, the implemented approach can be routinely applied for calculations of the tunneling splittings for the molecules and molecular clusters investigated with MW and MMW spectroscopy.

Supplementary Information The online version contains supplementary material available at https://doi.org/10.1007/s11224-021-01845-4.

Acknowledgements This research was supported in part through the Maxwell computational resources operated at Deutsches ElektronenSynchrotron DESY, Hamburg, Germany. D.S.T. would also like to thank Prof. Melanie Schnell for the helpful discussions and general support with this work. 
Author contributions D.S.T. has constructed the presented numerical approach, wrote the Python code, performed benchmarking, and wrote the manuscript.

Funding Open Access funding enabled and organized by Projekt DEAL.

Data availability The quantum-chemical calculations needed to reproduce the results, the results themselves, and raw data for the plots of this paper can be obtained online as Supplementary materials.

Code availability The Python scripts used for calculation of Hamiltonian parameters can be downloaded from the repository (https://stash. desy.de/projects/MOLINC) [74], the version used for calculations in this paper is also available from the SI.

\section{Declarations}

\section{Ethics approval Not applicable}

Consent to participate Not applicable

Consent for publication Not applicable

Conflicts of interest/Competing interests Conflicts of interest/Competing interests

Open Access This article is licensed under a Creative Commons Attribution 4.0 International License, which permits use, sharing, adaptation, distribution and reproduction in any medium or format, as long as you give appropriate credit to the original author(s) and the source, provide a link to the Creative Commons licence, and indicate if changes were made. The images or other third party material in this article are included in the article's Creative Commons licence, unless indicated otherwise in a credit line to the material. If material is not included in the article's Creative Commons licence and your intended use is not permitted by statutory regulation or exceeds the permitted use, you will need to obtain permission directly from the copyright holder. To view a copy of this licence, visit http://creativecommons.org/licenses/by/4.0/.

\section{References}

1. Giese K, Petkovic M, Naundorf H, Kühn O (2006) Multidimensional quantum dynamics and infrared spectroscopy of hydrogen bonds. Phys Rep 430(4):211-276

2. Leitner DM, Havenith M, Gruebele M (2006) Biomolecule large-amplitude motion and solvation dynamics: modelling and probes from thz to X-rays. Int Rev Phys Chem 25(4):553-582

3. Löwdin P-O (1963) Proton tunneling in dna and its biological implications. Rev Mod Phys 35:724-732

4. Srivastava R (2019) The role of proton transfer on mutations. Front Chem 7:536

5. Tikhonov DS, Rykov AN, Grikina OE, Khaikin LS (2016) Gas phase equilibrium structure of histamine. Phys Chem Chem Phys 18:6092-6102

6. Baba T, Tanaka T, Morino I, Yamada KMT, Tanaka K (1999) Detection of the tunneling-rotation transitions of malonaldehyde in the submillimeter-wave region. J Chem Phys 110(9):4131-4133

7. Baughcum SL, Duerst RW, Rowe WF, Smith Z, Wilson EB (1981) Microwave spectroscopic study of malonaldehyde (3-hydroxy-2-propenal) 2 structure, dipole moment, and tunneling. J Am Chem Soc 103(21):6296-6303

8. Baughcum SL, Smith Z, Wilson EB, Duerst RW (1984) Microwave spectroscopic study of malonaldehyde 3 vibration-rotation interaction and one-dimensional model for proton tunneling. J Am Chem Soc 106(8):2260-2265

9. Li W, Evangelisti L, Gou Q, Caminati W, Meyer R (2019) The barrier to proton transfer in the dimer of formic acid: A pure rotational study. Angew Chem Int Ed 58(3):859-865

10. Nguyen HVL, Kleiner I (2020) Understanding (coupled) large amplitude motions: the interplay of microwave spectroscopy, spectral modeling, and quantum chemistry. Phys Sci Rev

11. Pérez C, Muckle MT, Zaleski DP, Seifert NA, Temelso B, Shields GC, Kisiel Z, Pate BH (2012) Structures of cage, prism, and book isomers of water hexamer from broadband rotational spectroscopy. Science 336(6083):897-901

12. Špirko V, Kraemer W (1989) Anharmonic potential function and effective geometries for the nh3 molecule. J Mol Spectrosc 133(2):331-344

13. Pérez C, Steber AL, Rijs AM, Temelso B, Shields GC, Lopez JC, Kisiel Z, Schnell M (2017) Corannulene and its complex with water: a tiny cup of water. Phys Chem Chem Phys 19:14214-14223

14. Schnell M, Grabow J-U (2006) Multidimensional largeamplitude motion: Revealing concurrent tunneling pathways in molecules with several internal rotors. Angew Chem Int Ed 45(21):3465-3470

15. Zhang Y, Li W, Luo W, Zhu Y, Duan C (2017) High resolution jet-cooled infrared absorption spectra of (hcooh)2, (hcood)2, and hcooh-hcood complexes in $7.2 \mu \mathrm{m}$ region. J Chem Phys 146(24):244306

16. Fatima M, Maué D, Pérez C, Tikhonov DS, Bernhard D, Stamm A, Medcraft C, Gerhards M, Schnell M (2020) Structures and internal dynamics of diphenylether and its aggregates with water. Phys Chem Chem Phys 22:27966-27978

17. Saragi RT, Juanes M, Pérez C, Pinacho P, Tikhonov DS, Caminati W, Schnell M, Lesarri A (2021) Switching hydrogen bonding to $\pi$-stacking: The thiophenol dimer and trimer. J Phys Chem Lett 12(5):1367-1373

18. Liu H, Cao J, Bian W (2019) Double proton transfer in the dimer of formic acid: An efficient quantum mechanical scheme. Front Chem 7:676

19. Marechal Y, Witkowski A (1968) Infrared spectra of h-bonded systems. J Chem Phys 48(8):3697-3705

20. Birer Ö, Havenith M (2009) High-resolution infrared spectroscopy of the formic acid dimer. Annu Rev Phys Chem 60(1):263-275 (PMID: 18999993)

21. Ortlieb M, Havenith M (2007) Proton transfer in (hcooh)2: An ir high-resolution spectroscopic study of the antisymmetric c-o stretch. J Phys Chem A 111(31):7355-7363 (PMID: 17552500)

22. Babic D, Bosanac S, Došlic N (2002) Proton transfer in malonaldehyde: a model three-dimensional study. Chem Phys Lett 358(3):337-343

23. Matanović I, Došlić N, Johnson BR (2008) Generalized approximation to the reaction path: The formic acid dimer case. J Chem Phys 128(8):084103

24. Vener MV, Kühn O, Bowman JM (2001) Vibrational spectrum of the formic acid dimer in the oh stretch region a model $3 \mathrm{~d}$ study. Chem Phys Lett 349(5):562-570

25. Barnes GL, Sibert EL (2008) The effects of asymmetric motions on the tunneling splittings in formic acid dimer. J Chem Phys 129(16): 164317

26. Barnes GL, Squires SM, Sibert EL (2008) Symmetric double proton tunneling in formic acid dimer: A diabatic basis approach. $\mathrm{J}$ Phys Chem B 112(2):595-603 (PMID: 18004835) 
27. Tew DP, Handy NC, Carter S (2006) A reaction surface hamiltonian study of malonaldehyde. J Chem Phys 125(8):084313

28. Yang Y, Meuwly M (2010) A generalized reactive force field for nonlinear hydrogen bonds: Hydrogen dynamics and transfer in malonaldehyde. J Chem Phys 133(6):064503

29. Coutinho-Neto M, Viel A, Manthe U (2004) The ground state tunneling splitting of malonaldehyde: Accurate full dimensional quantum dynamics calculations. J Chem Phys 121(19):9207-9210

30. Hammer T, Manthe $U$ (2011) Intramolecular proton transfer in malonaldehyde: Accurate multilayer multi-configurational timedependent hartree calculations. J Chem Phys 134(22):224305

31. Hayashi T, Mukamel S (2003) Multidimensional infrared signatures of intramolecular hydrogen bonding in malonaldehyde. $\mathrm{J}$ Phys Chem A 107(43):9113-9131

32. Qu C, Bowman JM (2016) An ab initio potential energy surface for the formic acid dimer: zero-point energy, selected anharmonic fundamental energies, and ground-state tunneling splitting calculated in relaxed 14-mode subspaces. Phys Chem Chem Phys 18:24835-24840

33. Wu F, Ren Y, Bian W (2016) The hydrogen tunneling splitting in malonaldehyde: A full-dimensional time-independent quantum mechanical method. J Chem Phys 145(7):074309

34. Viel A, Coutinho-Neto MD, Manthe U (2007) The ground state tunneling splitting and the zero point energy of malonaldehyde: A quantum monte carlo determination. J Chem Phys 126(2):024308

35. Wang Y, Braams BJ, Bowman JM, Carter S, Tew DP (2008) Fulldimensional quantum calculations of ground-state tunneling splitting of malonaldehyde using an accurate $a b$ initio potential energy surface. J Chem Phys 128(22):224314

36. Mil'nikov G, Nakamura H (2008) Tunneling splitting and decay of metastable states in polyatomic molecules: invariant instanton theory. Phys Chem Chem Phys 10:1374-1393

37. Mil'nikov GV, Kühn O, Nakamura H (2005) Ground-state and vibrationally assisted tunneling in the formic acid dimer. J Chem Phys 123(7):074308

38. Richardson JO (2017) Full- and reduced-dimensionality instanton calculations of the tunnelling splitting in the formic acid dimer. Phys Chem Chem Phys 19:966-970

39. Richardson JO, Althorpe SC (2011) Ring-polymer instanton method for calculating tunneling splittings. J Chem Phys 134(5):054109

40. Smedarchina Z, Fernandez-Ramos A, Siebrand W (2004) Calculation of the tunneling splitting in the zero-point level and co-stretch fundamental of the formic acid dimer. Chem Phys Lett 395(4):339-345

41. Kochikov I, Tarasov Y, Vogt N, Spiridonov V (2002) Largeamplitude motion in 1,4-cyclohexadiene and 1,4-dioxin: theoretical background for joint treatment of spectroscopic, electron diffraction and ab initio data. J Mol Struct 607(2):163-174

42. Kochikov IV, Tarasov YI (2003) Equilibrium structure and internal rotation in b2f4 from electron diffraction and spectroscopic data and quantum chemical calculations. Struct Chem 14(2):227-238

43. Neese F (2012) The orca program system. Wiley Interdisciplinary Reviews: Computational Molecular Science 2(1):73-78

44. Perdew JP, Burke K, Ernzerhof M (1997) Generalized gradient approximation made simple [phys. rev. lett. 77, 3865 (1996)]. Phys Rev Lett 78:1396

45. Adamo C, Barone V (1999) Toward reliable density functional methods without adjustable parameters: The pbe 0 model. J Chem Phys 110(13):6158-6170

46. Becke AD (1988) Density-functional exchange-energy approximation with correct asymptotic behavior. Phys Rev A 38:3098-3100

47. Becke AD (1993) Density-functional thermochemistry iii the role of exact exchange. J Chem Phys 98(7):5648-5652

48. Lee C, Yang W, Parr RG (1988) Development of the colle-salvetti correlation-energy formula into a functional of the electron density. Phys Rev B 37:785-789
49. Yanai T, Tew DP, Handy NC (2004) A new hybrid exchangecorrelation functional using the coulomb-attenuating method (cam-b3lyp). Chem Phys Lett 393(1):51-57

50. Grimme S (2006) Semiempirical hybrid density functional with perturbative second-order correlation. J Chem Phys 124(3):034108

51. Schwabe T, Grimme S (2006) Towards chemical accuracy for the thermodynamics of large molecules: new hybrid density functionals including non-local correlation effects. Phys Chem Chem Phys 8:4398-4401

52. Bartlett RJ, Musiał M (2007) Coupled-cluster theory in quantum chemistry. Rev Mod Phys 79:291-352

53. Grimme S (2003) Improved second-order moller-plesset perturbation theory by separate scaling of parallel- and antiparallelspin pair correlation energies. J Chem Phys 118(20):9095-9102

54. Møller C, Plesset MS (1934) Note on an approximation treatment for many-electron systems. Phys Rev 46(7):618-622

55. Grimme S, Ehrlich S, Goerigk L (2011) Effect of the damping function in dispersion corrected density functional theory. J Comput Chem 32(7):1456-1465

56. Dunlap BI, Connolly JWD, Sabin JR (1979) On some approximations in applications of $x \alpha$ theory. J Chem Phys 71(8):3396-3402

57. Izsák R, Neese F (2011) An overlap fitted chain of spheres exchange method. J Chem Phys 135(14):144105

58. Kossmann S, Neese F (2009) Comparison of two efficient approximate hartee-fock approaches. Chem Phys Lett 481(4):240-243

59. Kossmann S, Neese F (2010) Efficient structure optimization with second-order many-body perturbation theory: The rijcosxmp2 method. J Chem Theory Comput 6(8):2325-2338 (PMID: 26613489)

60. Neese F (2003) An improvement of the resolution of the identity approximation for the formation of the coulomb matrix. J Comput Chem 24(14):1740-1747

61. Neese F, Wennmohs F, Hansen A, Becker U (2009) Efficient, approximate and parallel hartree-fock and hybrid dft calculations a 'chain-of-spheres' algorithm for the hartree-fock exchange. Chem Phys 356(1):98-109

62. Whitten JL (1973) Coulombic potential energy integrals and approximations. J Chem Phys 58(10):4496-4501

63. Hellweg A, Hättig C, Höfener S, Klopper W (2007) Optimized accurate auxiliary basis sets for ri-mp2 and ri-cc2 calculations for the atoms rb to rn. Theor Chem Accounts 117(4):587-597

64. Weigend $\mathrm{F}$ (2006) Accurate coulomb-fitting basis sets for $\mathrm{h}$ to $\mathrm{rn}$. Phys Chem Chem Phys 8:1057-1065

65. Weigend $\mathrm{F}$ (2008) Hartree-fock exchange fitting basis sets for $\mathrm{h}$ to rn. J Comput Chem 29(2):167-175

66. Weigend F, Ahlrichs R (2005) Balanced basis sets of split valence, triple zeta valence and quadruple zeta valence quality for $h$ to rn: Design and assessment of accuracy. Phys Chem Chem Phys 7:3297-3305

67. Carrington T, Miller WH (1984) Reaction surface hamiltonian for the dynamics of reactions in polyatomic systems. J Chem Phys 81(9):3942-3950

68. Carrington T, Miller WH (1986) Reaction surface description of intramolecular hydrogen atom transfer in malonaldehyde. J Chem Phys 84(8):4364-4370

69. Miller WH, Handy NC, Adams JE (1980) Reaction path hamiltonian for polyatomic molecules. J Chem Phys 72(1):99-112

70. Shida N, Barbara PF, Almlöf J (1991) A reaction surface hamiltonian treatment of the double proton transfer of formic acid dimer. J Chem Phys 94(5):3633-3643

71. Meyer R, Günthard HH (1968) General internal motion of molecules, classical and quantum-mechanical hamiltonian. J Chem Phys 49(4):1510-1520

72. Wilson E, Decius J, Cross P (1980) Molecular Vibrations: The Theory of Infrared and Raman Vibrational Spectra. Dover Books on Chemistry Series, Dover Publications 
73. Ochterski JW (1999) Vibrational analysis in gaussian

74. Tikhonov DS (2020) https://stash.desy.de/projects/MOLINC

75. Colbert DT, Miller WH (1992) A novel discrete variable representation for quantum mechanical reactive scattering via the s-matrix kohn method. J Chem Phys 96(3):1982-1991

76. Perdew JP, Schmidt K (2001) Jacob's ladder of density functional approximations for the exchange-correlation energy. AIP Conf Proc 577(1): 1-20

77. Dohm S, Hansen A, Steinmetz M, Grimme S, Checinski MP (2018) Comprehensive thermochemical benchmark set of realistic closed-shell metal organic reactions. J Chem Theory Comput 14(5):2596-2608 (PMID: 29565586)

78. Kraus P (2020) Basis set extrapolations for density functional theory. J Chem Theory Comput 16(9):5712-5722 (PMID: 32790303)

Publisher's Note Springer Nature remains neutral with regard to jurisdictional claims in published maps and institutional affiliations. 